\title{
Speckle Imaging Over Horizontal Paths
}

\author{
C. J. Carrano
}

This article was submitted to High-Resolution Wavefront Control: Methods, Devices and Applications IV (AM102), Seattle, Washington, July $7-11,2002$

\section{May 21, 2002}

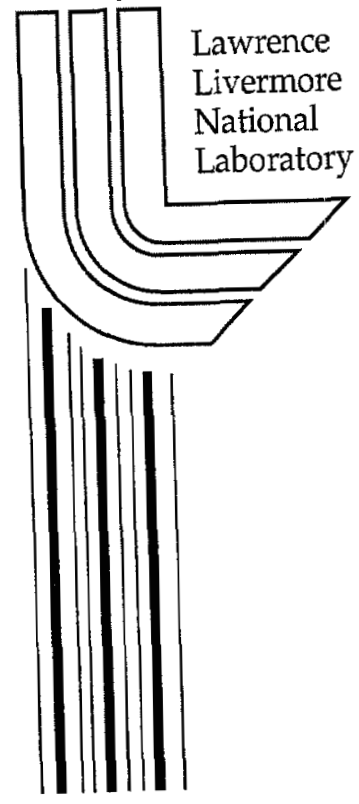




\section{DISCLAIMER}

This document was prepared as an account of work sponsored by an agency of the United States Government. Neither the United States Government nor the University of California nor any of their employees, makes any warranty, express or implied, or assumes any legal liability or responsibility for the accuracy, completeness, or usefulness of any information, apparatus, product, or process disclosed, or represents that its use would not infringe privately owned rights. Reference herein to any specific commercial product, process, or service by trade name, trademark, manufacturer, or otherwise, does not necessarily constitute or imply its endorsement, recommendation, or favoring by the United States Government or the University of California. The views and opinions of authors expressed herein do not necessarily state or reflect those of the United States Government or the University of California, and shall not be used for advertising or product endorsement purposes.

This is a preprint of a paper intended for publication in a journal or proceedings. Since changes may be made before publication, this preprint is made available with the understanding that it will not be cited or reproduced without the permission of the author.

This work was performed under the auspices of the United States Department of Energy by the University of California, Lawrence Livermore National Laboratory under contract No. W-7405-Eng-48.

This report has been reproduced directly from the best available copy.

Available electronically at http://www.doc.gov/bridge

Available for a processing fee to U.S. Department of Energy And its contractors in paper from

U.S. Department of Energy

Office of Scientific and Technical Information

P.O. Box 62

Oak Ridge, TN 37831-0062

Telephone: (865) 576-8401

Facsimile: (865) 576-5728

E-mail: reports@adonis.osti.gov

Available for the sale to the public from

U.S. Department of Commerce

National Technical Information Service

5285 Port Royal Road

Springfield, VA 22161

Telephone: (800) 553-6847

Facsimile: (703) 605-6900

E-mail: orders@ntis.fedworld.gov

Online ordering: http://www.ntis.gov/ordering.htm

OR

Lawrence Livermore National Laboratory

Technical Information Department's Digital Library

http://www.llnl.gov/tid/Library.html 


\title{
Speckle imaging over horizontal paths
}

\author{
Carmen J. Carrano* \\ Lawrence Livermore National Laboratory, 7000 East Avenue, Livermore, CA 94550
}

\begin{abstract}
Atmospheric aberrations reduce the resolution and contrast in surveillance images recorded over horizontal or slant paths. This paper describes our recent horizontal and slant path imaging experiments of extended scenes as well as the results obtained using speckle imaging. The experiments were performed with an 8-inch diameter telescope placed on either a rooftop or hillside and cover ranges of interest from $0.5 \mathrm{~km}$ up to $10 \mathrm{~km}$. The scenery includes resolution targets, people, vehicles, and other structures. The improvement in image quality using speckle imaging is dramatic in many cases, and depends significantly upon the atmospheric conditions. We quantify resolution improvement through modulation transfer function measurement comparisons.
\end{abstract}

Keywords: horizontal-path imaging, slant-path imaging, bispectral speckle imaging, extended scene reconstruction

\section{INTRODUCTION}

Bispectral speckle imaging techniques have been used for many years to correct atmospheric effects in astronomical ${ }^{1,2}$ and satellite imagery ${ }^{3}$ approaching diffraction-limited resolution. The common feature between these applications is that the telescope is pointing up through the atmosphere and the object being imaged is typically smaller than the isoplanatic angle. In this paper we will investigate the performance of a bispectral imaging algorithm when the telescope images extended scenes through a horizontal or slant path through the atmosphere.

\section{EXPERIMENTAL DESCRIPTION}

\subsection{Equipment}

Our prototype system for data acquisition is quite simple and consists of three primary parts: telescope, camera, and computer. The telescope is a Celestron 8-inch diameter Schmidt-Cassegrain with a focal length of $2032 \mathrm{~mm}$ and a 2 inch central obscuration. The camera is a Qimaging Retiga 1300, which is a 12-bit CCD camera with 1280 by 1024 pixels whose size is 6.7 by $6.7 \mu \mathrm{m}$. The camera has a readout noise level of $8 \mathrm{e}$ - and is capable of exposure times down to $40 \mu \mathrm{s}$. In order to satisfy Nyquist sampling at our center wavelength of $550 \mathrm{~nm}$, we use a $3 x$ Barlow lens between the telescope and camera. With the 3x Barlow lens included, the angular pixel size is $1.1 \mu$ radians. Control of the camera as well as data acquisition is done with a PC laptop through an IEEE1394 (firewire) connection. At full camera resolution, we are able to acquire images at a few frames per second. Once the data is acquired, processing can be done on the laptop or on a faster $\mathrm{PC}$.

\subsection{Experiments}

Experiments were performed from one of two locations, either the rooftop of a two story building onsite or from a hillside 500 feet above a road looking out at various distances. Figure 1 shows the hillside telescope location along with the target locations.

\footnotetext{
"E-mail: carrano2@1lnl.gov
} 


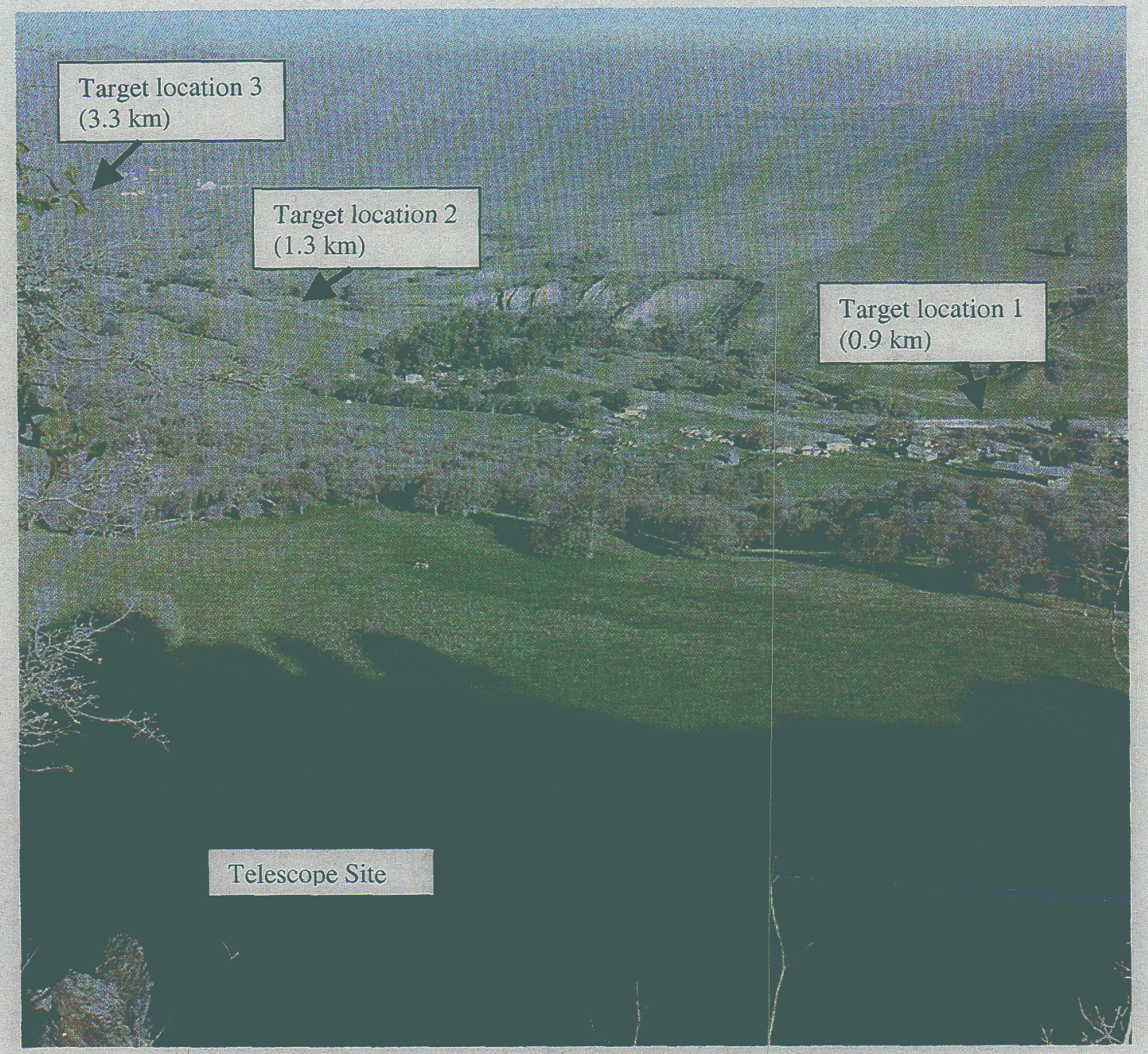

Figure 1: Digital camera picture showing hillside telescope site and the target locations. A person or people and resolution targets were placed at each of the three target locations, $0.9,1.3$, and $3.3 \mathrm{~km}$ distance. The telescope site is at an altitude of 500 feet (152 meters) above the target locations.

\section{IMAGE PROCESSING}

Using our bispectral speckle image reconstruction software developed for satellite ${ }^{3}$ and astronomical ${ }^{4,5}$ imaging as a starting point, modifications needed to be made for the horizontal-path case. Issues to be addressed included accommodating extended scenery, smaller isoplanatic patches, new data formats, and increased data sizes. But before getting into the new issues, let us summarize the basic speckle imaging concepts.

\subsection{Review of speckle imaging}

A much more detailed description of our original speckle imaging techniques can be found in Ref. 6 , but for completeness, an overview is included here. The purpose of speckle imaging is to obtain a diffraction-limited estimate of the true object from a time series of short exposure images of the object. This is done by estimating both the Fourier magnitude and phase of the object, independently, and then inverse transforming. Figure 2 summarizes the processing steps. 


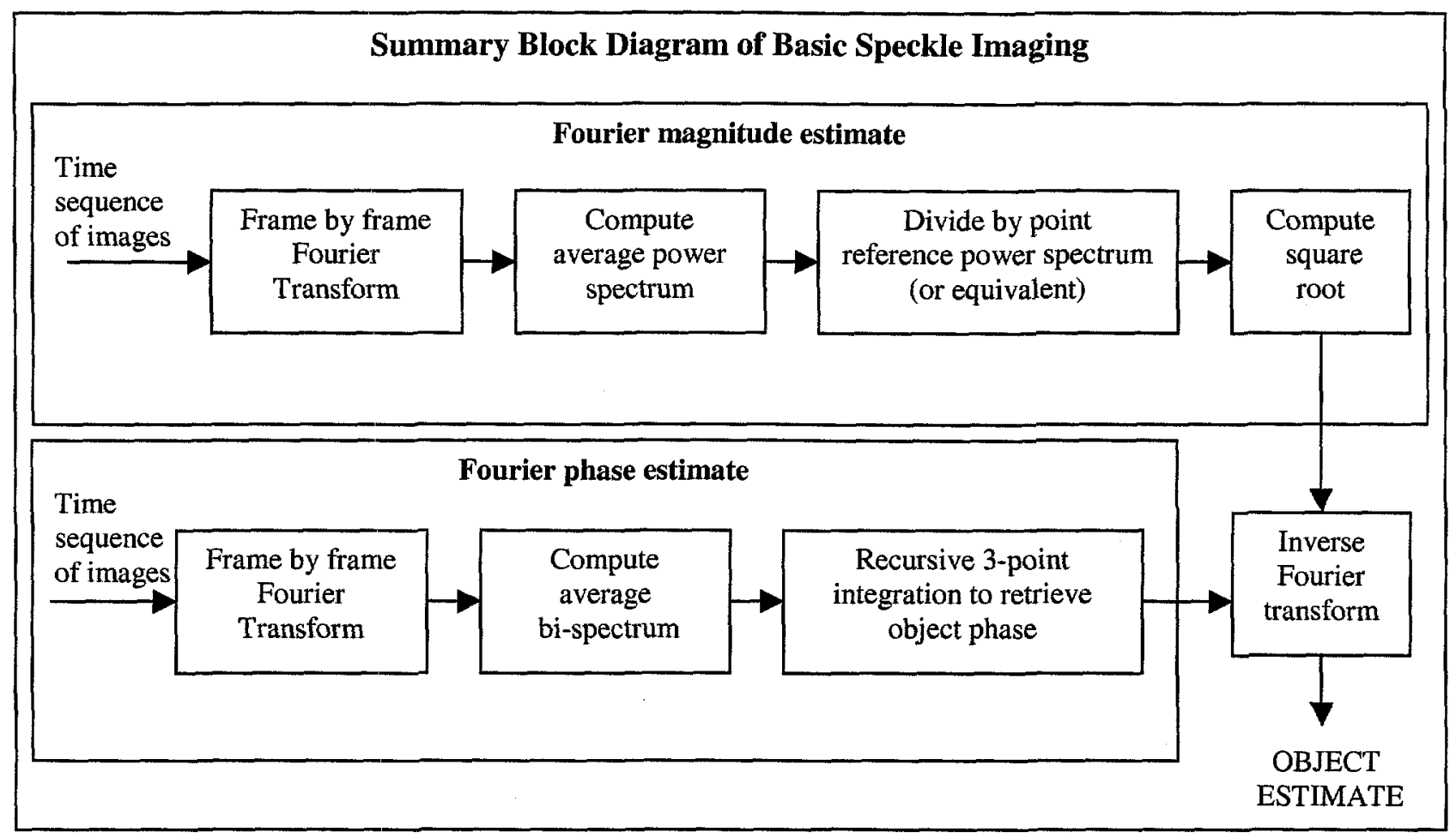

Figure 2: Block diagram summarizing the processing steps for basic bispectral speckle imaging.

We can model the imaging with the following convolution:

$$
i_{n}(\mathbf{x})=\tau_{n}(\mathbf{x}) * o(\mathbf{x}),
$$

where $i_{n}(\mathbf{x})$ is the nth speckle image in an ensemble, $o(\mathbf{x})$ is the object we want to recover, and $\tau_{n}(\mathbf{x})$ is the combined atmosphere-telescope point-spread function. If we Fourier transform this relationship, the convolution becomes a multiplication. We can then average the powerspectrum over each frame and solve for the Fourier magnitude.

$$
|O(\mathbf{u})|_{\text {est }}=\left[\frac{\left\langle\left|I_{n}(\mathbf{u})\right|^{2}\right\rangle_{n}}{\left\langle\left|\tau_{n}(\mathbf{u})\right|^{2}\right\rangle_{n}}\right]^{1 / 2}
$$

Because we are dealing with horizontal path imagery, we most likely do not have a point reference for generating $\left\langle\left|\tau_{n}(\mathbf{x})\right|^{2}\right\rangle$. Instead, we use a model for this, where $r_{0}$, the atmospheric coherence length, is chosen and plugged into the Labeyrie-Korff transfer function ${ }^{7}$. Since we don't typically know $r_{0}$, we iterate to find the best value. Trying values of $r_{0}$ between $1 \mathrm{~cm}$ and $4 \mathrm{~cm}$ in increments of $0.5 \mathrm{~cm}$ will nearly always yield one or more acceptable images.

For the phase estimate, we make use of the complex bispectrum. The bispectrum is defined in spatial frequency space as:

$$
I_{B, n}(\mathbf{u}, \mathbf{v}) \equiv I_{n}(\mathbf{u}) I_{n}(\mathbf{v}) I_{n}(-\mathbf{u}-\mathbf{v})
$$

where $u$ and $v$ are spatial frequency vectors. It is shown in Ref. 6 that the Fourier phase of the object is recursively related to the phase of the average complex bispectrum according to a three-point integration:

$$
\arg |O(\mathbf{u}+\mathbf{v})|=\arg |O(\mathbf{u})|+\arg |O(\mathbf{v})|-\arg \left|\left\langle I_{B, n}(\mathbf{u}, \mathbf{v})\right\rangle_{n}\right|
$$


Starting with aribtrary boundary conditions at the origin and the first two frequency points on axis in the frequency domain, it is possible to recover the object phase through recursive application of Equation 4 . In fact, since there are many combinations of $u$ and $v$ that give the same $u+v$, we can average over large amounts of non-redundant information. Our implementation of this recursion allows us to choose how much averaging 9is performed. Values between 4 and 8 averaged data points give nice results.

After the Fourier magnitude and phase are estimated, they are simply combined and inverse transformed.

\subsection{Additions to basic algorithm}

In this section, we discuss additional processing steps needed prior to and after the speckle processing itself. A block diagram outlining the steps is given in Figure 3.

In the first step, once we have converted the raw camera data, which are individual 16-bit TIFF files, to a 3-D datacube containing a sequence 2-D images, we need to account for any bad pixels or groups of pixels that were obscured by dust on the optics. We can locate these pixels by acquiring an image of the sky or a severely out of focus image of low contrast scenery and thresholding the data to give us a map. Once we have the map, the bad pixels of each frame of the image sequence are replaced by the average of the surrounding good pixels.

In the second step, we perform a global frame by frame registration. In the data acquisition, both telescope shaking and atmospheric tip/tilt contribute to $\mathrm{x}$ and $\mathrm{y}$ shifting of the frames. Shifts are calculated using the standard Fourier correlation methods ${ }^{8}$ on high-passed versions of the data. We usually take the first frame as the alignment reference, assuming it is a representative frame. One could also align to the frame average just as easily. The shifting is typically less than ten percent of the full image size, which means that the outer pixels of the processed image will be unreliable. The need for frame registration will become clear as we describe subsequent processing steps.

An intrinsic feature of horizontal-path imaging through volume turbulence is that the isoplanatic angles will typically be much smaller than the scene you need to image. As in solar astronomy ${ }^{9}$, we have found that breaking up the image sequence into small regions or tiles can significantly improve the reconstructed image quality. In this processing step, the $1280 x 1024 \mathrm{x} \#$ frames datacube is split up into a number of smaller overlapped 3-D datacubes. The size of the tiles we use is typically $256 \times 256$ pixels with $50 \%$ overlapping. For a $1280 \times 1024$ image, it means processing $9 \times 7$ or 63 tiles. While in solar astronomy, choice of tile size is directly related to the isoplanatic patch size ${ }^{\text {, }}$, we are finding the optimal tile size to be somewhat related to the object being imaged combined with the amount of apodization that is required. Determining the optimal tile size is still under investigation.

Unlike astronomical scenes, where the object of interest is compact and the data essentially falls away to zero outside the object, horizontal-path terrestrial scenery fill the image. This means that prior to the Fourier transform step of speckle processing, windowing or edge apodization will be needed in order to avoid ringing at the edges. In order to preserve as much of the scene as possible, our choice of window leaves the center of the scene untouched and tapers the edges of the scene. We have found that a Hanning window taper works well for most cases. See Figure 4 for an example window. The amount of apodization that should be used, which determines the steepness or slope of the window taper, depends on the atmospheric conditions. If the blurring caused by the atmosphere covers $\mathrm{N}$ pixels, then the window taper needs to span greater than $\mathrm{N}$ pixels. If the taper is too sharp, the speckle processing puts artifacts in the results. We have found apodization between $50 \%$ and $80 \%$ works quite well.

After each tile is processed, the full image is assembled. Since the tiles overlap by $50 \%$, we apply a standard square-shaped Hanning window to each reconstructed tile and add them up in the proper locations. Hanning windows have the nice feature that when shifted by half a cycle and summed, they add to one. This means there are no artifacts from the overlapping and adding of the tiles.

Currently, processing out to the diffraction limit for a $256 \times 256 \times 100$ sized datacube on a single processor $1.7 \mathrm{GHz}$ Pentium IV takes approximately 20 seconds using a bispectral averaging depth of 8 and without any attempts to optimize the software. With some software and hardware modifications, the processing times could be decreased to a few seconds per sub-field or less. 


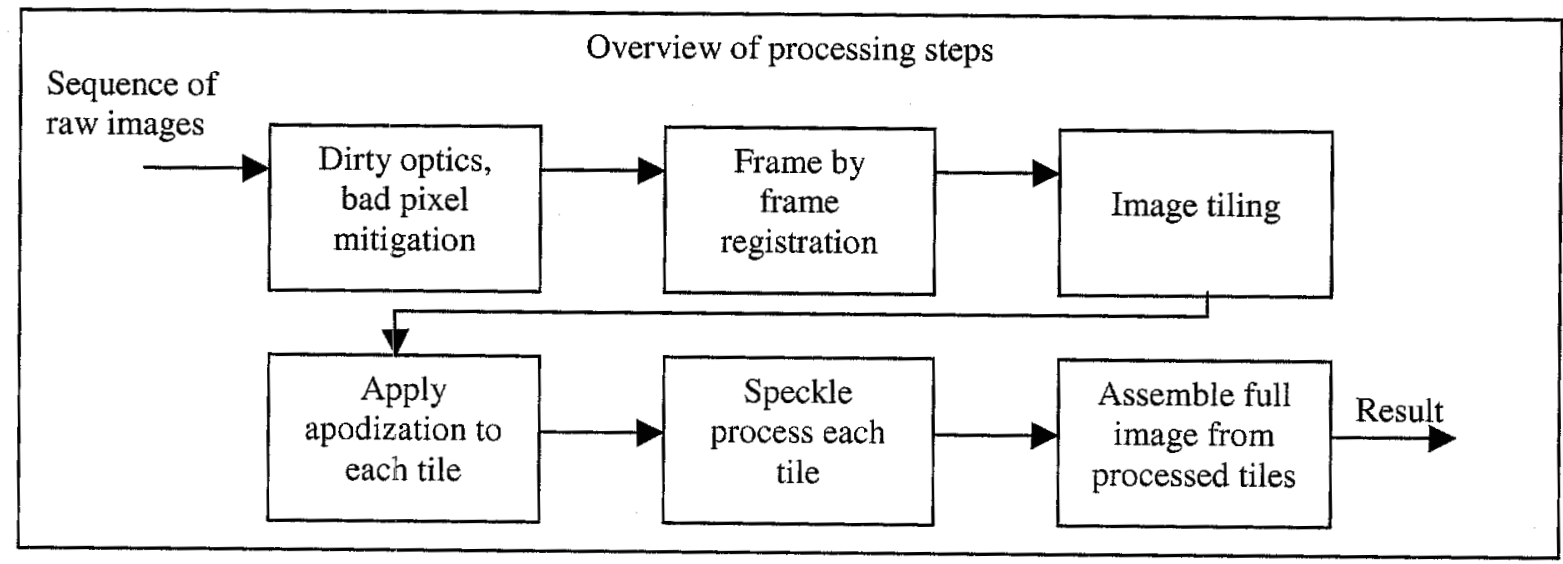

Figure 3: Block diagram overview of processing steps for producing enhanced images of extended scene, horizontal path imagery via speckle processing.

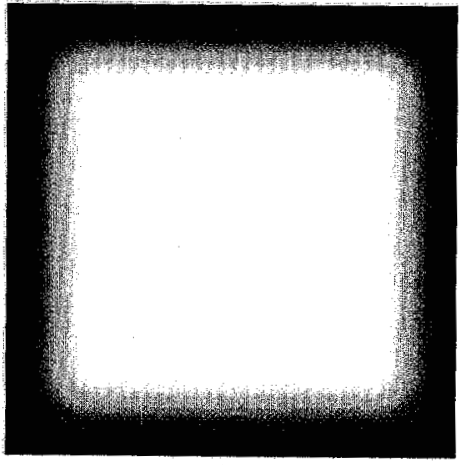

(a.)

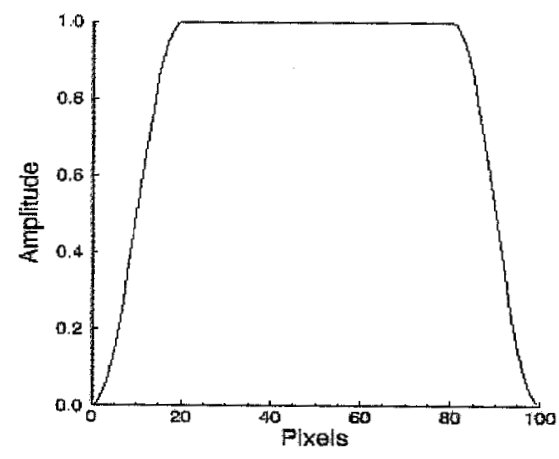

(b.)

Figure 4: Example of $60 \%$ apodization with Hanning window taper with (a.) 2D image of window and (b.) lineout through the window.

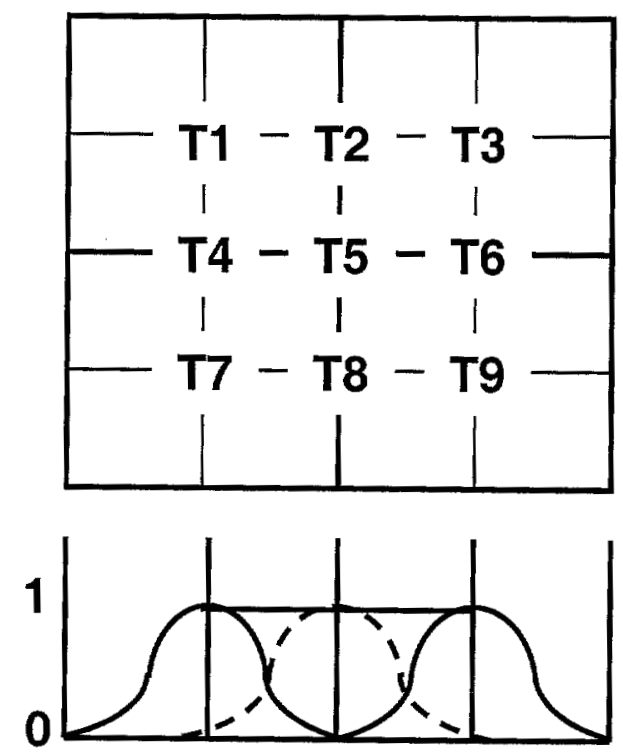

Figure 5: Example of how tiles (T1-T9) are assembled to form the full field image using Hanning window weighting. 


\section{RESULTS}

We have done a number of experiments to establish the performance of the extended-scene, horizontal-path speckle processing techniques described in the previous section. We now look at some experimental results from a variety of targets and ranges.

The first result we show is from point source target at $0.5 \mathrm{~km}$. The point source was placed in a field, while the telescope was looking across the field from the roof of a two story building. The data was taken near sunset, when atmospheric perturbations are slightly lower. With 50 frames and an exposure time of $2 \mathrm{~ms}$, we obtain a diffraction limited reconstruction as seen in Figure 6 . The $r_{0}$ used for this case was $3.0 \mathrm{~cm}$

The next result we show (Figure 7) is for a black and white fan target, with known frequency content. The target was constructed by printing the fan pattern with a laser printer on standard white $8.5 \times 11$ inch paper and mounting it on a larger board. It was placed at $0.5 \mathrm{~km}$ range. For this dataset, we acquired 50 frames at a $5 \mathrm{~ms}$ exposure time per frame on a sunny, windy day during the summer. In Figure 7 we show two example frames, a shift and add result, as well as full-field and sub-field speckle processing. The tile size used in this case was 512x512 pixels with $60 \%$ Hanning apodization, and $\mathrm{r}_{0}$ was set to $1.25 \mathrm{~cm}$. Both speckle processed images show dramatic improvement in resolution and contrast over the raw frames or the shift and add image, but the one with tiling has somewhat better resolution, fewer artifacts, and it more accurately represents the true object (Figure 7f). The diffraction limit is $1.4 \mathrm{~mm}$ for a $20 \mathrm{~cm}$ diameter telescope at $0.5 \mathrm{~km}$ range and $550 \mathrm{~nm}$ wavelength and is where we would expect the modulation to go to zero. The reconstructed image with tiling shows ungarbled modulation all the way down to the diffraction limit.

The next few datasets we show were taken from the telescope site as pictured in Figure 1. At target location \#1, a person stood $0.9 \mathrm{~km}$ away from the telescope, while we acquired 100 frames at $10 \mathrm{~ms}$ exposure time per frame. The data was taken midday during the winter on a partly sunny, somewhat calm day. In Figure 8 we show example frames, the shift and add image, full-field and sub-field speckle processed results. The tile size for the sub-field results was $256 \times 256$ pixels with $60 \%$ Hanning apodization with $\mathrm{r}_{0}$ set to $2.0 \mathrm{~cm}$. Again, both speckle processed images are dramatically better than unprocessed frames or the shift and add image, but the tile processed image is slightly better, both in resolution and accuracy in representing the object. In fact, the quality of the tile processed image is such that face recognition becomes possible both qualitatively and with commercial facial recognition software.

The next set of results is for the same person placed at $1.3 \mathrm{~km}$ range, which is target location \#2 as pictured in Figure 1. In Figure 9 we show an example short exposure frame, the shift and add result, full-field and sub-field speckle processing results. We acquired 100 frames of data with an exposure time of $10 \mathrm{~ms}$ per frame. The sub-field result was obtained using a tile size of $256 \times 256$ pixels with $60 \%$ Hanning apodization, and $r_{0}$ set to $2.0 \mathrm{~cm}$. The tile processed image is clearly the best resolution image. The word "MAGELLAN" on the box, whose letters are $1.6 \mathrm{~cm}$ tall, is clearly readable and the face is recognizable. In the analysis section we will show MTF results from our resolution target at this same site.
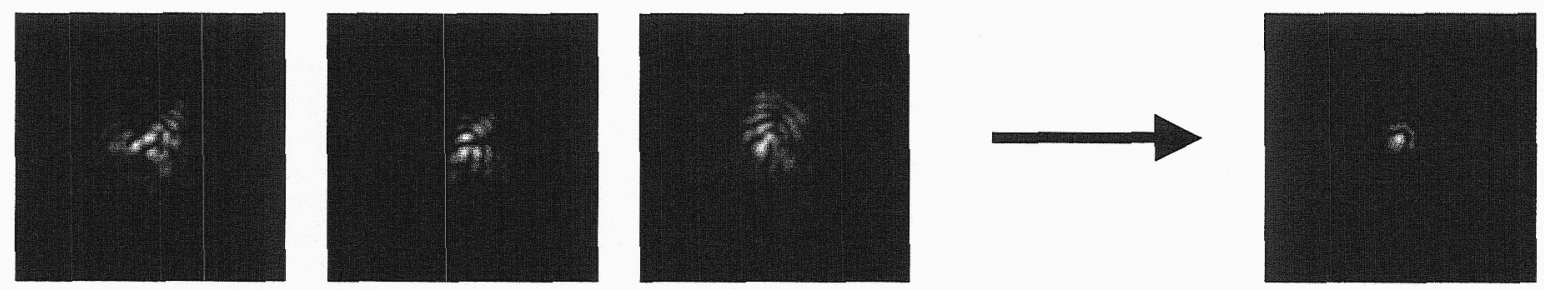

Figure 6: Shown in this figure are example raw frames (left 3 images) with an exposure time of $2 \mathrm{~ms}$ and the speckle processed result(right) of a sub-resolution point target. 


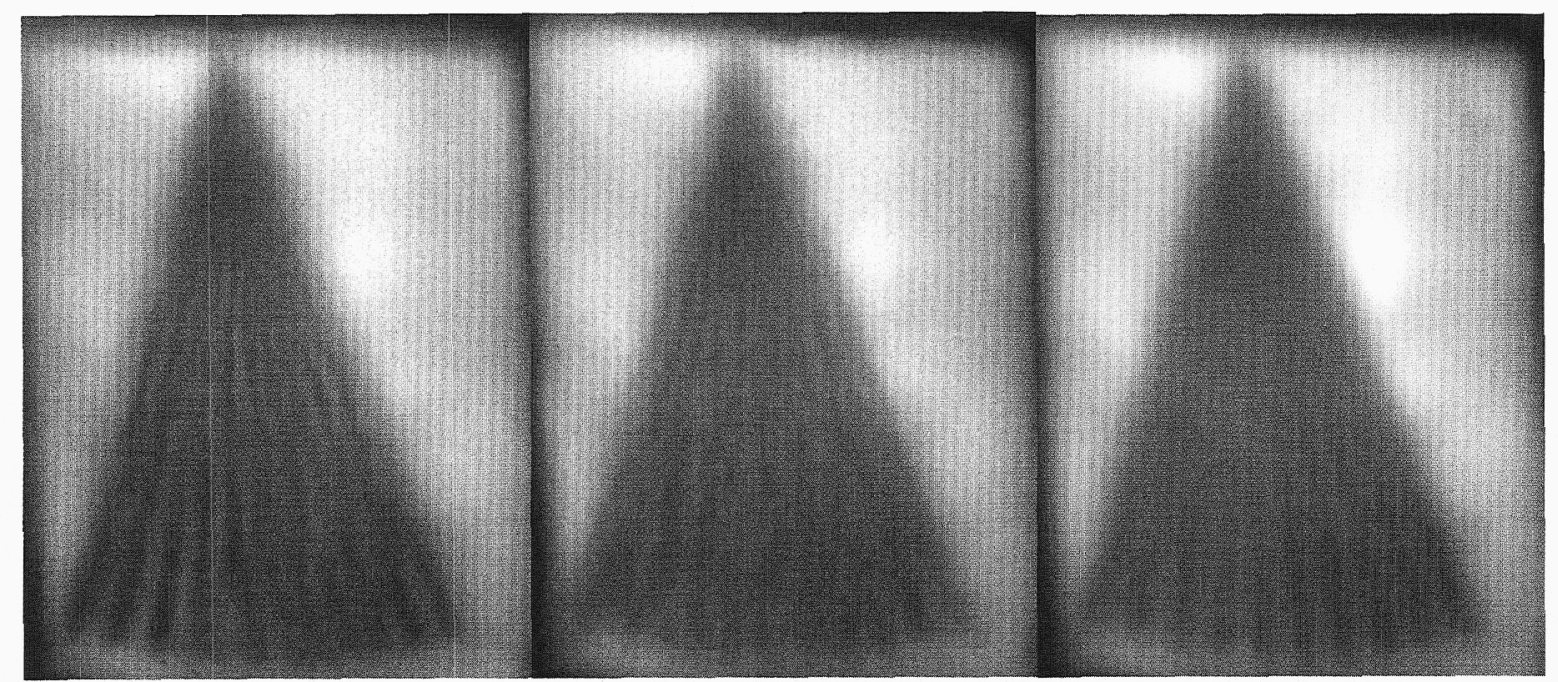

a. Example raw frame

b. Example raw frame

c. Shift and add 50 frames

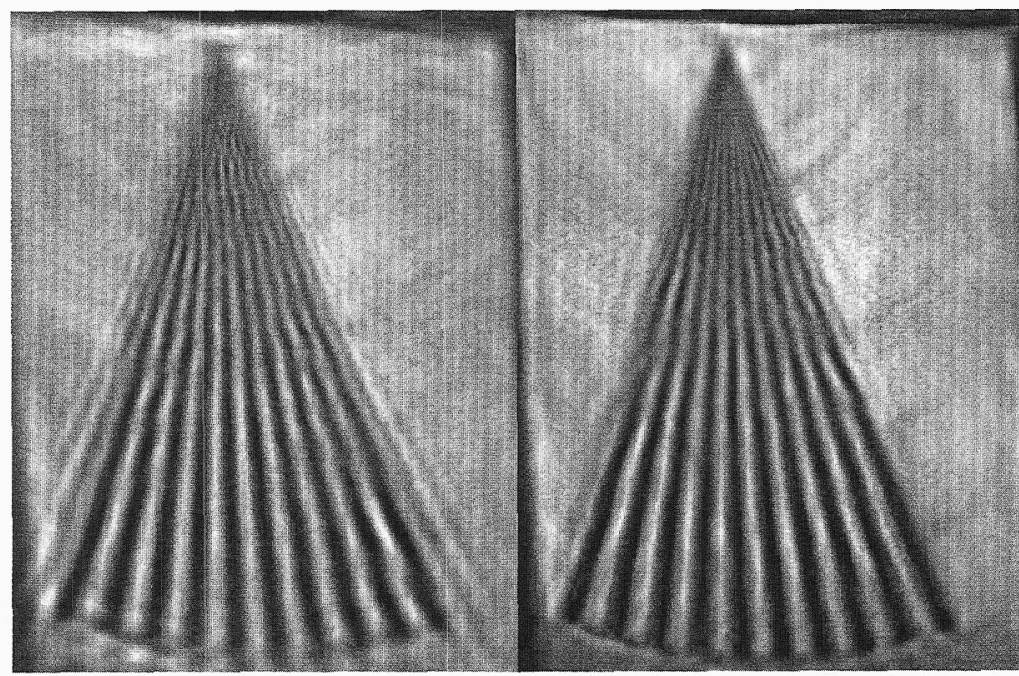

d. Full field speckle processing e. Sub-field speckle processing

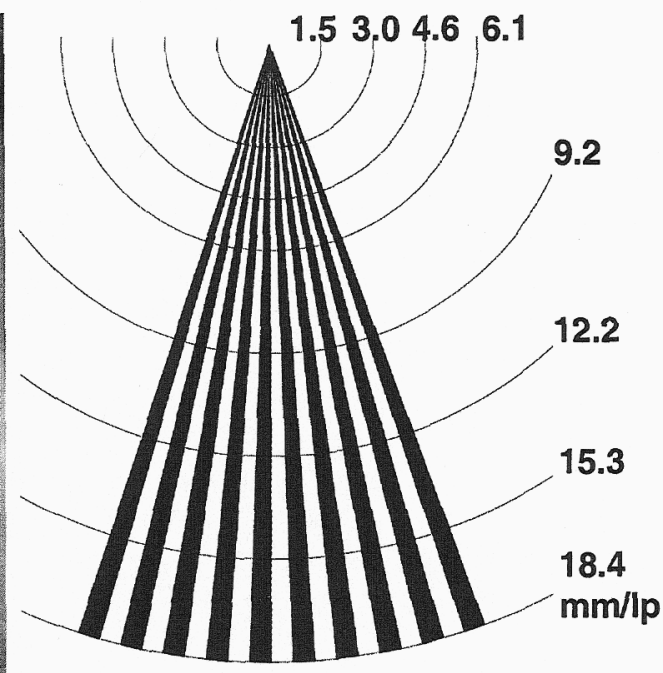

f. Ground truth for fan target

Figure 7: Results from fan target imaged at $0.5 \mathrm{~km}$ range with $5 \mathrm{~ms}$ exposure time. 


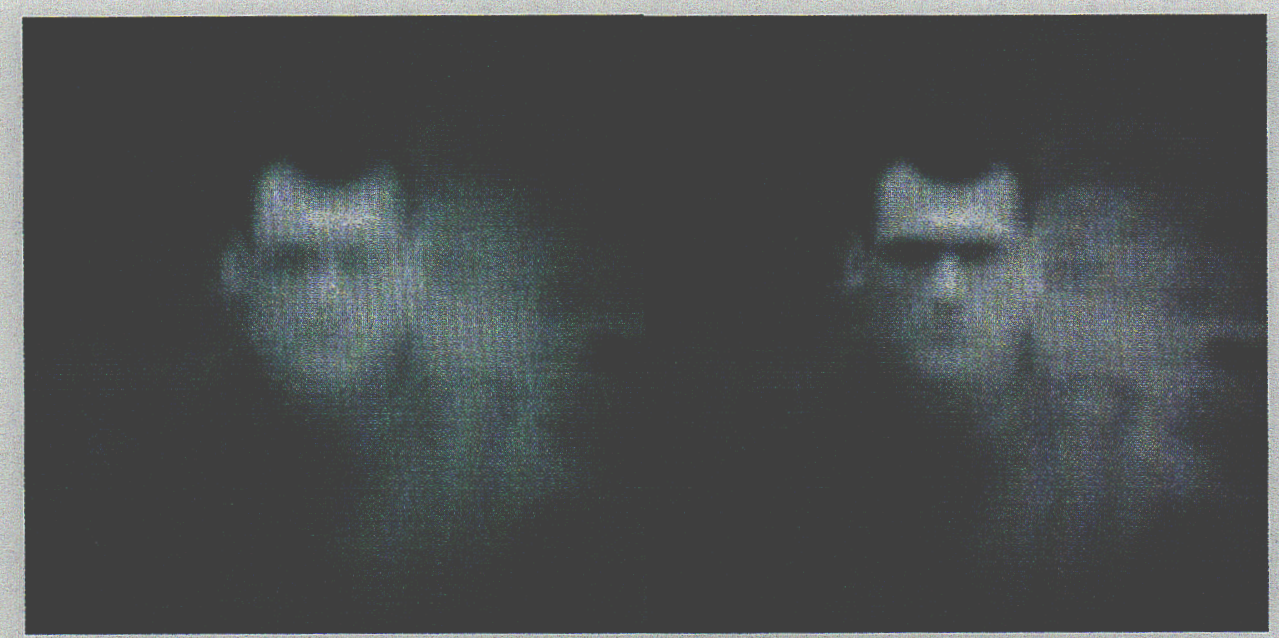

a. Example of a more blurry frame

b. Example of a less blurry frame

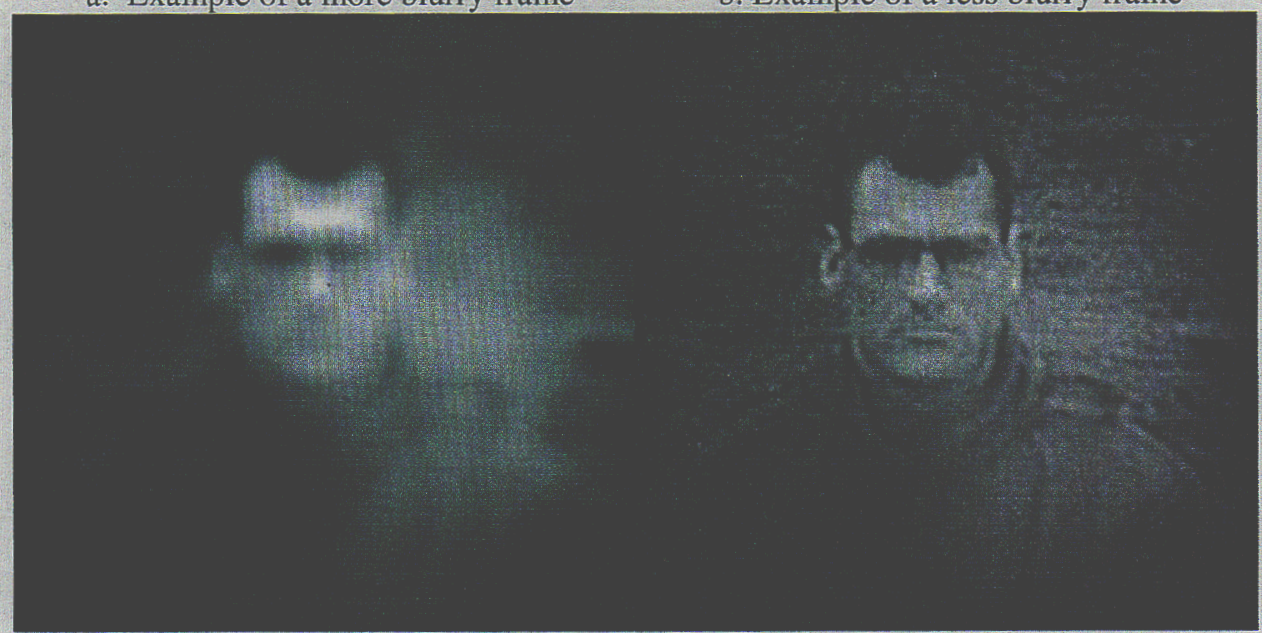

c. Shift and add 100 frames

d. Full- field speckle processing

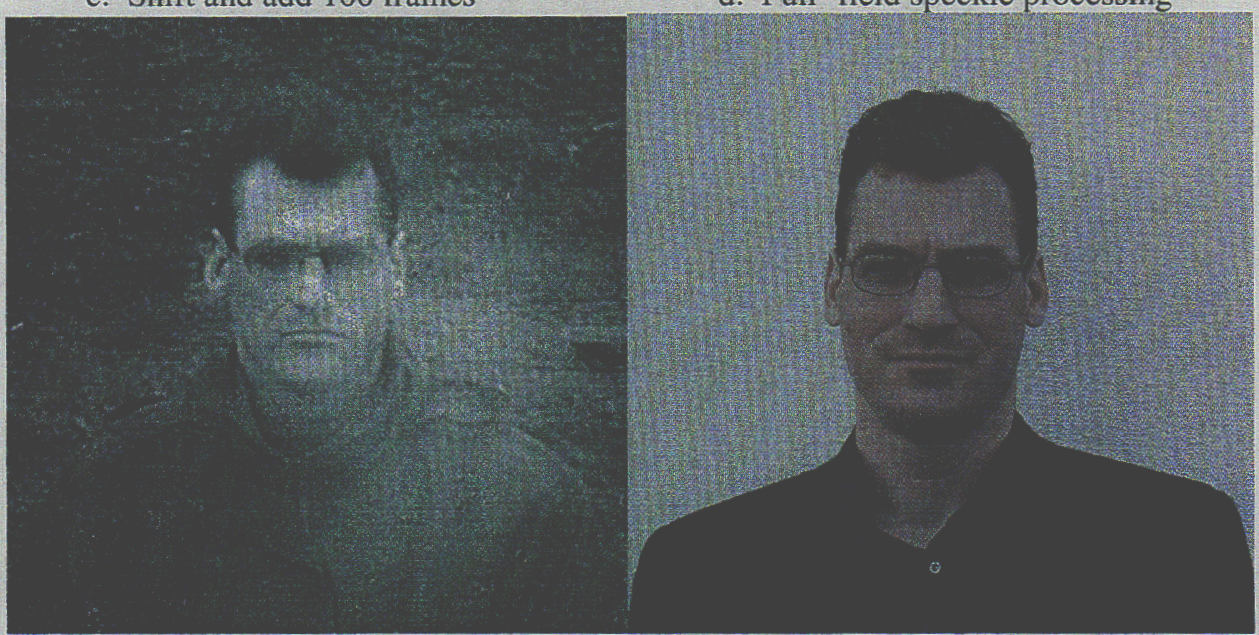

e. Sub-field speckle processing

f. Photograph of our subject

Figure 8: Results of a person imaged at $0.9 \mathrm{~km}$ distance. The center $900 \times 900$ pixels are displayed in each case. The sub-field speckle processed result is with tiles of $256 \times 256$ pixels. 


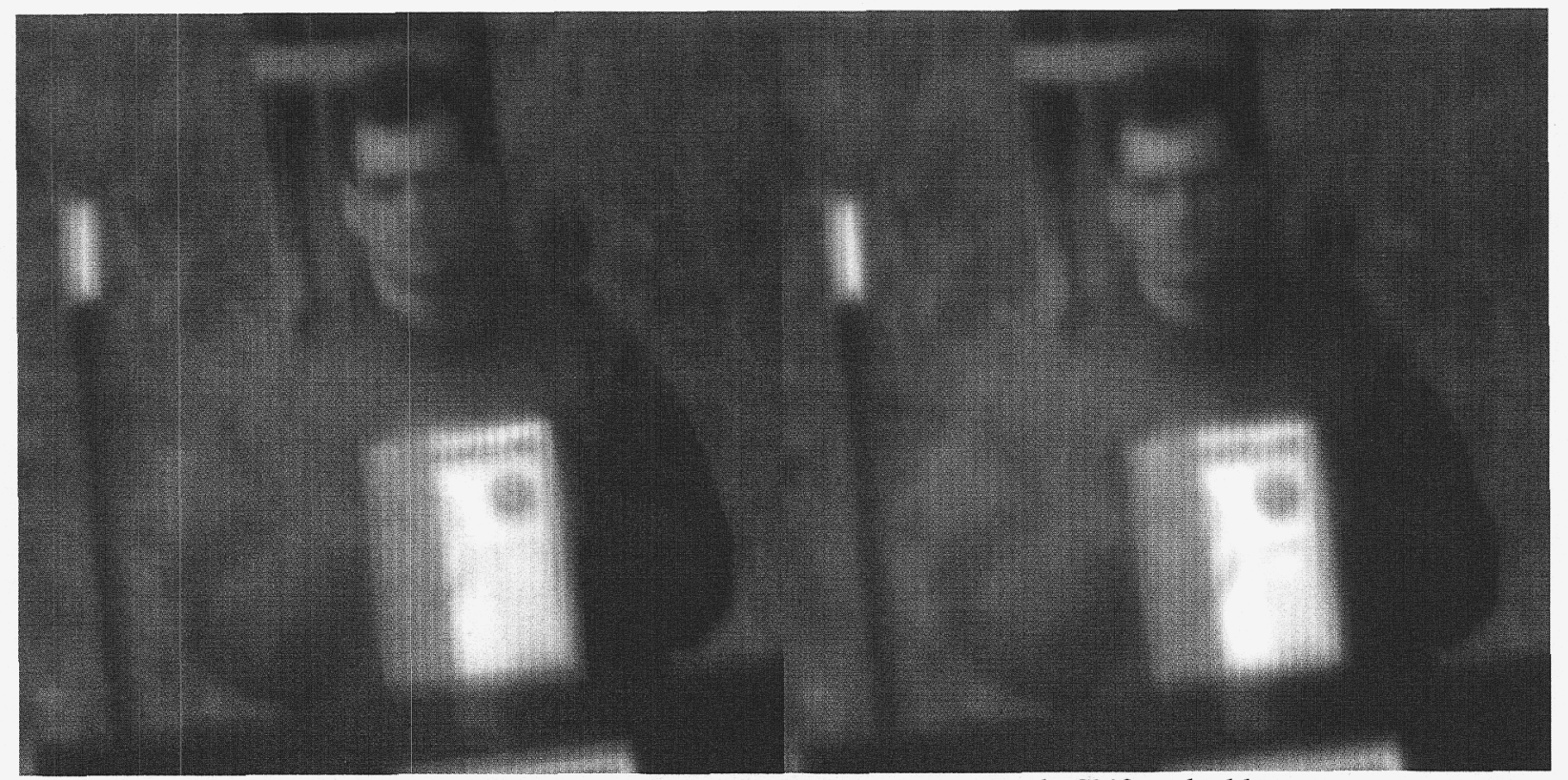

a. Example frame

b. Shift and add

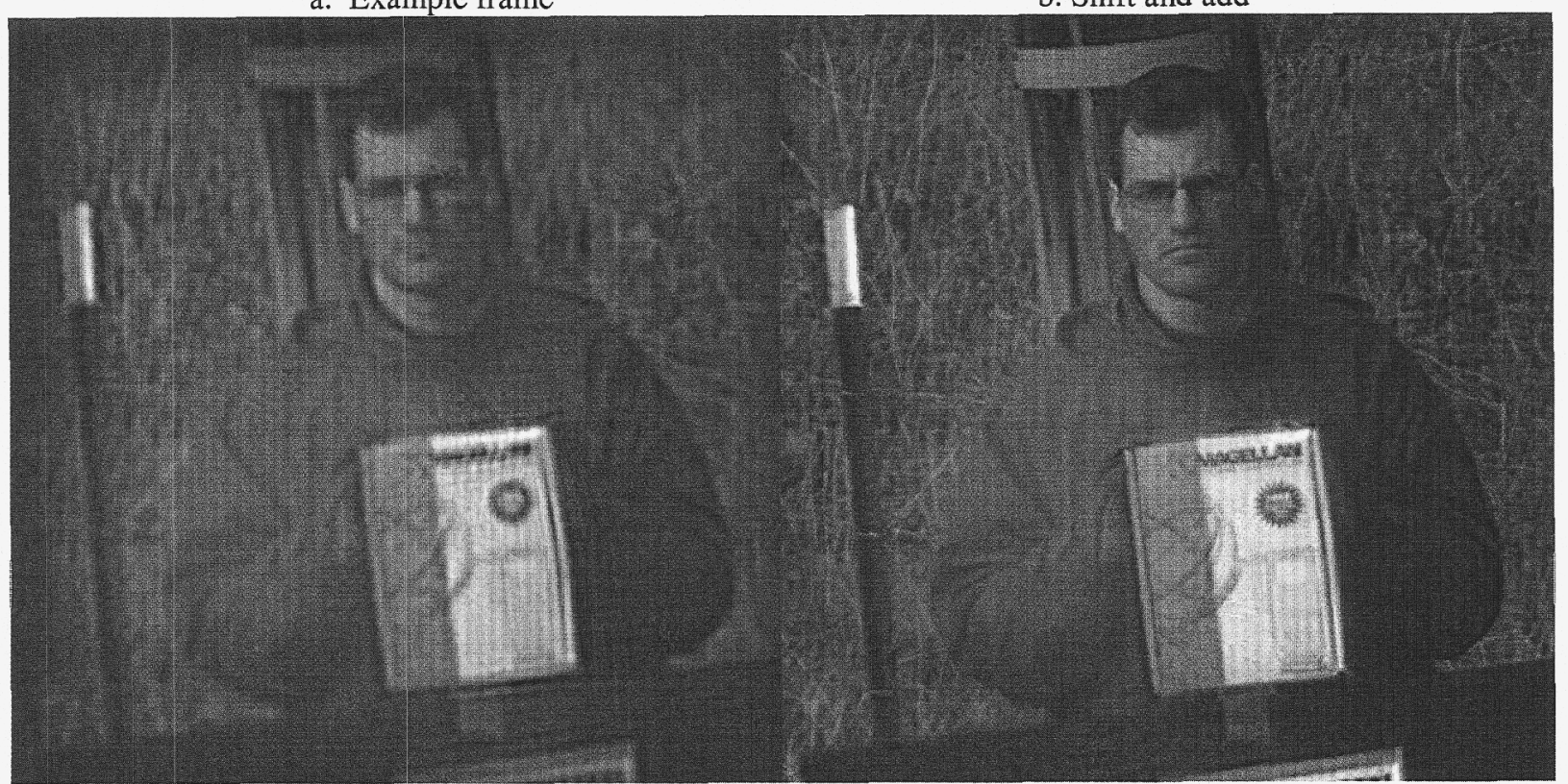

c. Full field processing

d. Sub-field processing

Figure 9: Results of person imaged at $1.3 \mathrm{~km}$.

This next dataset we show is of two people and a resolution target at $3.3 \mathrm{~km}$ distance, (target location \#3 in Figure 1.) It was taken on a different day from the previous two datasets. This dataset was acquired on a warm and breezy spring-like day with varied terrain below the imaging path, including grass, trees, and asphalt. We acquired 100 frames with $8 \mathrm{~ms}$ exposure time per frame. The shift and add image and the sub-field speckle processed result are shown in Figure 10. The tile size used for the speckle processing was $256 \times 256$ pixel s with $60 \%$ apodization. At $3.3 \mathrm{~km}$ and $550 \mathrm{~nm}$, the diffraction limited resolution is about $1 \mathrm{~cm}$. In the images, it is possible to see that we are approaching 
the diffraction limit, in that finger and facial features are observable, and the $1.3 \mathrm{~cm}$ wide white border surrounding the four resolution patterns clearly visible.

Imaging from our two-story rooftop on a sunny and very windy day we acquired 100 frames of the front of a semi-truck at $9 \mathrm{~km}$ range. Exposure time was $8 \mathrm{~ms}$ per frame. Figure 11 shows the comparison between the shift and add image and the speckle processed image using $512 \times 512$ sized tiles and an $r_{0}$ of $1.2 \mathrm{~cm}$. While there are some artifacts in the reconstructed image and the performance is not quite diffraction limited, it is most certainly higher resolution than a single frame or the shift and add image.

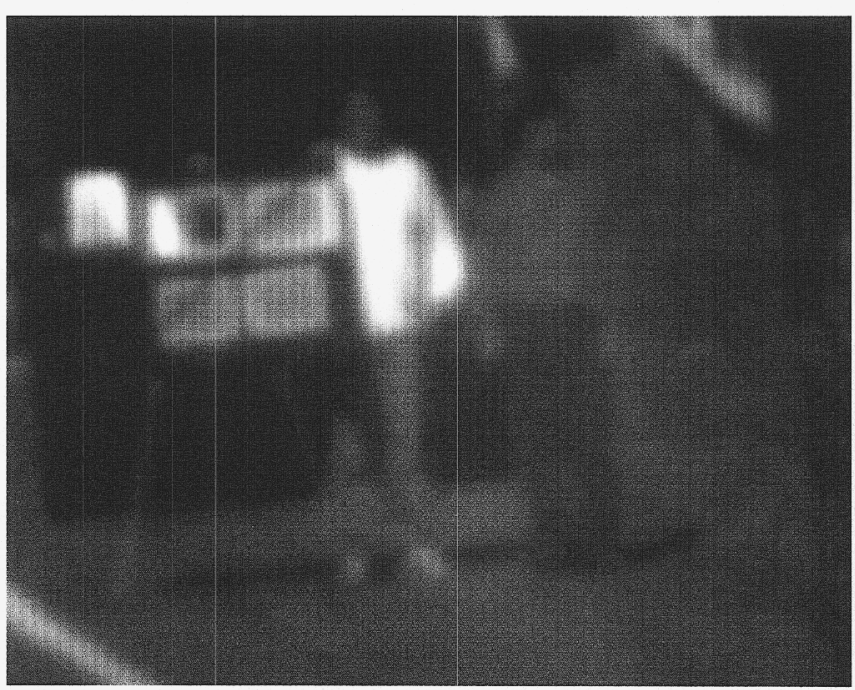

a. Shift and add

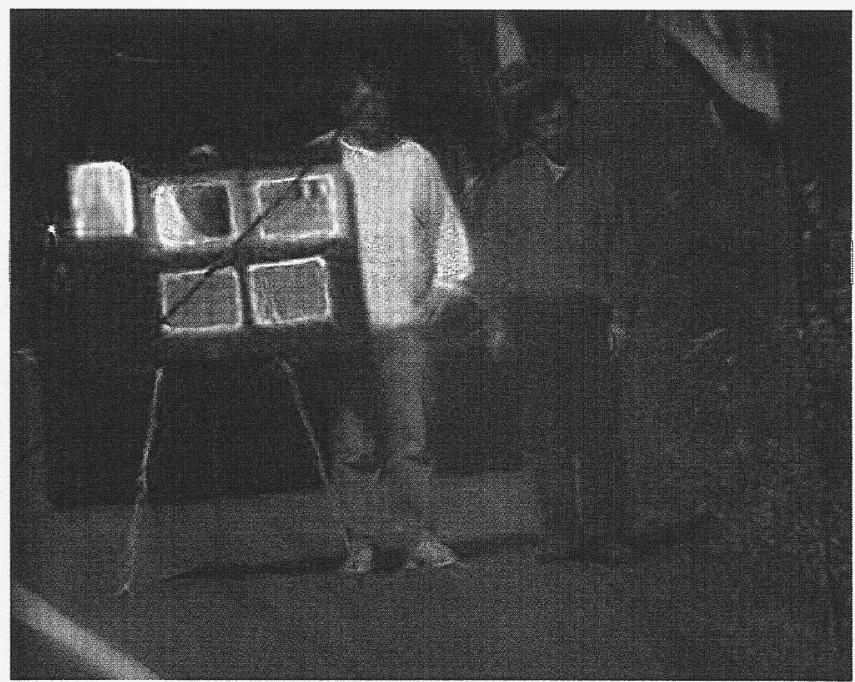

b. Speckle processed image

Figure 10: Result of people and resolution targets at $3.3 \mathrm{~km}$ range.

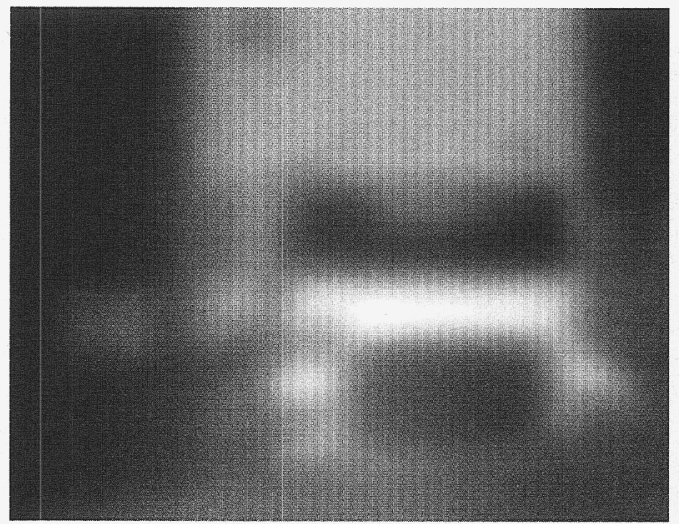

a. Shift and add image

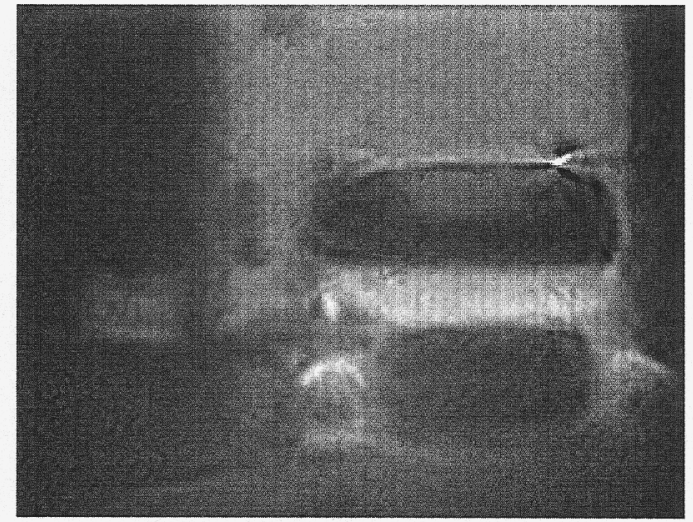

b. Speckle processed image

Figure 11: Result of semi-truck at $9 \mathrm{~km}$ range.

\section{ANALYSIS}

A common method for analyzing the performance of an imaging system is to measure its modulation transfer function (MTF). MTF is a measure of how well spatial details are preserved over a range of frequencies, typically from zero 
frequency up to the diffraction limit of the system. In order to measure this function we constructed a resolution target with sine wave patterns on it at varying frequencies of interest. By measuring the modulation in the resulting images for each of the frequency samples we are able to construct a curve and compare it to the curve for an ideal telescope. Modulation is defined as:

$$
\text { Modulation }=\frac{\left(I_{\max }-I_{\min }\right)}{\left(I_{\max }+I_{\min }\right)} .
$$

Normalization for the measured modulation was performed by dividing the observed modulation for a very low frequency case, essentially DC. The case we will consider for MTF analysis is for 100 frames of our resolution target as imaged from $1.3 \mathrm{~km}$ with a $10 \mathrm{~ms}$ exposure time. In Figure 12, we see the shift and add image and the speckle processed image using 256x256 pixel sized tiles. In Figure 13 we compare the ideal MTF curve for our telescope with that from the shift and add image and the speckle processed image. It is clear both in the image and in the MTF plot that the speckle processing improves the image resolution nearly to the diffraction limit. Performance at the lower frequencies $(<0.1 \mathrm{lp} / \mathrm{mm})$ is somewhat adjustable by choice of $r_{0}$ in the deconvolution step. The data shown here is for an $r_{0}$ of 4.0 $\mathrm{cm}$.

\section{CONCLUSIONS}

We have demonstrated the use of bispectral speckle imaging for use in correcting atmospheric blurring in surveillance images recorded over long horizontal paths. Sub-field processing is used to obtain better results than could be obtained processing the full field. The results with personnel at ranges between a few hundred meters up to a few kilometers are such that long distance personnel identification is possible.

We are continuing work in this area to better understand the relationship between the actual atmospheric profile and the achievable image quality.

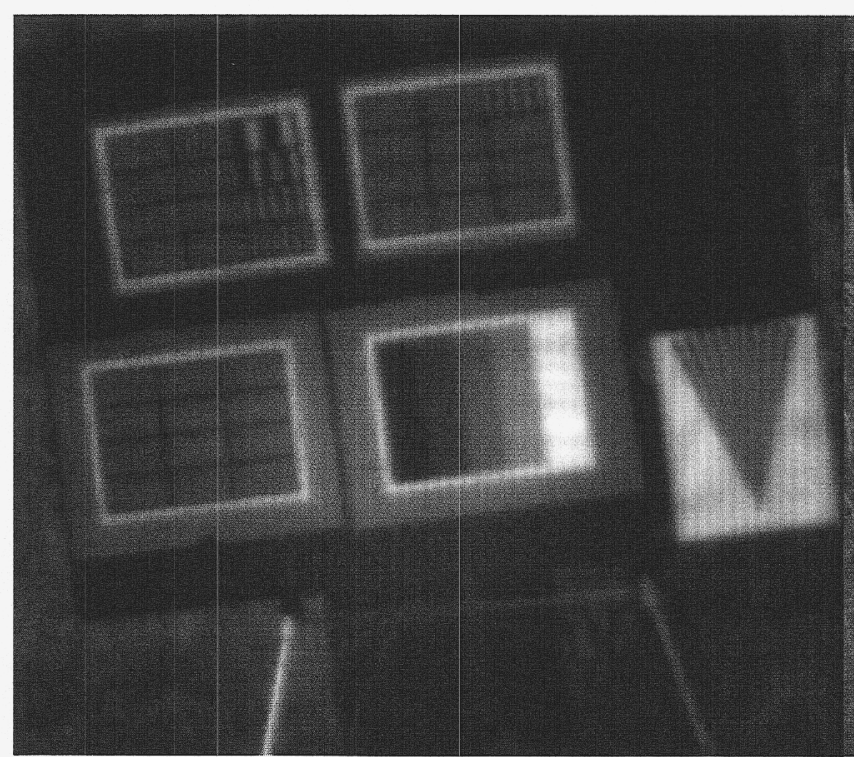

a. Shift and add image

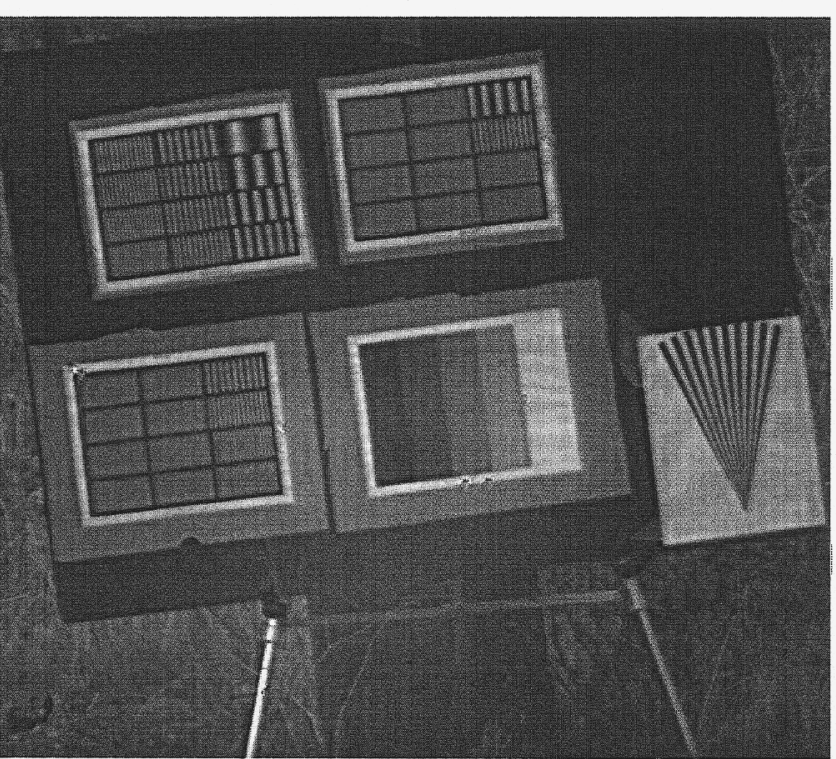

b. Speckle processed image

Figure 12: Results for resolution target imaged at $1.3 \mathrm{~km}$ range. 


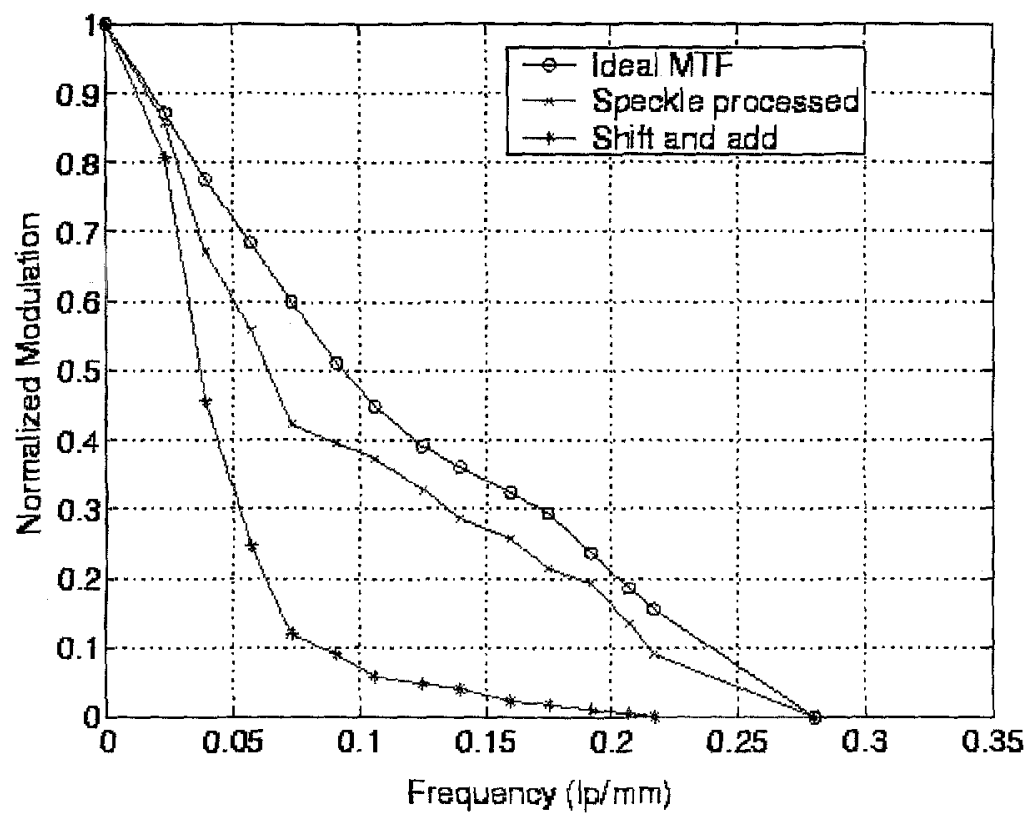

Figure 13: Comparison of MTF's for the shift and add image and the speckle processed image with the ideal curve for the telescope.

\section{ACKNOWLEDGEMENTS}

I would like to acknowledge the help of Kevin Baker for nearly all the experiments as well as the participation of Scott Wilks and Peter Young. I would also like to thank Scot Olivier, Kevin Baker, and Kai LaFortune for being willing imaging subjects.

Work performed under the auspices of the U.S. Department of Energy by the Lawrence Livermore National Laboratory under contract No. W-7405-ENG-48.

\section{REFERENCES}

1. A. W. Lohmann, G. Weigelt, and B. Wirnitzer, "Speckle masking in astronomy: triple correlation theory and applications," Appl. Opt. 22, 4028-4037 (1983)

2. H. Bartelt, A. W. Lohmann, and B. Wirnitzer, "Phase and amplitude recovery from bispectra," Appl. Opt. 23, 3121$3129(1984)$

3. T. W. Lawrence, D. M. Goodman, E. M. Johansson, and J.P. Fitch, "Speckle imaging of satellites at the U.S. Air Force Maui Optical Station", Appl. Opt. 31, 6307-6321 (1992)

4. D. T. Gavel, C. E. Max, E. J. Johansson, B. Sheerwood, M. Liu, B. Bradford, "Observations of Comet P/ShoemakerLevy 9 Impact on Jupiter from Lick Observatory Using a High Resolution Speckle Imaging Camera", IAU Symposium 156, Space Telescope Science Institute, Baltimore, MD, May 9-12, 1995

5. T. W. Lawrence, J.P. Fitch, D. M. Goodman, N. A. Massie, R. J. Sherwood, and E. M. Johansson, "Extended-image reconstruction through horizontal path turbulence using bispectral speckle interferometry," Opt. Eng. 32, 627-636 (1992)

6. S. G. Gibbard, B. Macintosh, D. Gavel, et. al., "Titan: High-Resolution Speckle Images from the Keck Telescope", Icarus, 139, 189-201 (1999)

7. D. Korff, "Analysis of a methods for obtaining near-diffraction limited information in the presence of atmospheric turbulence," J. Opt. Soc. Am. 63, 971-980 (1973)

8. L. G. Brown, "A Survey of Image Registration Techniques", ACM Computing Surveys, 24, 325-376

9. O. von der Luhe, "Speckle imaging of solar small scale structure: I. Methods", Astron. Astrophys. 268, 374-390 (1993) 